\title{
Preoperative Discharge Assessment Tool (PDAT): Predicting Disposition After Lumbar Spine Fusion
}

\section{Taryn E LeRoy ${ }^{1}$, Andrew Mason ${ }^{2}$, David Tybor ${ }^{3}$, Jonna Capecci ${ }^{4}$, Louis Jenis $^{4}$ and Ashley Rogerson ${ }^{1 *}$}

${ }^{1}$ Department of Orthopaedic Surgery, Tufts Medical Center, Boston, MA, USA

${ }^{2}$ University of Minnesota Medical Center, Minneapolis, MN, USA

${ }^{3}$ Tufts University School of Medicine, Boston, MA, USA

${ }^{4}$ Department of Orthopaedic Surgery, Mass General Brigham - Newton Wellesley

Hospital, Newton, MA, USA

*Corresponding Author: Ashley Rogerson, Department of Orthopaedic Surgery,

Mass General Brigham - Newton Wellesley Hospital, Newton, MA, USA.
Received: July 06, 2021

Published: July 20, 2021

(C) All rights are reserved by Ashley

Rogerson., et al.

\section{Abstract}

Background: Various forms of scoring tools have been utilized to predict discharge disposition after joint arthroplasty surgery but there is limited data following spine surgery. The aim of this study was to develop a Risk Assessment and Predictor Tool (RAPT) for patients undergoing lumbar fusion surgery to preoperatively assess patient disposition and coordinate discharge after lumbar spine fusion surgery.

Methods: Retrospective review of 300 patients undergoing lumbar spine fusion surgery at a single center from January 1, 2014 - December 31, 2014. Patient demographics, intraoperative, and postoperative data were collected. Patients discharged to an inpatient rehabilitation facility or skilled nursing facility were compared to those discharged home to determine which variables were significantly different between the groups. Variables with the highest predictive accuracy were used to develop the Preoperative Discharge Assessment Tool (PDAT).

Results: A total of 300 patients were analyzed of which 227 (76\%) were discharged directly home and 73 (24\%) were discharged to a rehab facility. The mean and standard deviation (SD) length of stay (LOS) was $3 \pm 1$ days for all patients. Variables included in the final scoring tool were American Society of Anesthesiologists physical status classification (ASA) classification, number of levels fused, home living situation, and preoperative assistive device (AD) use. Overall predictive accuracy of the PDAT was 85.5\%. Fifteen patients preferred to be discharged to rehab and $14(93.3 \%)$ of those patients were ultimately discharged to rehab. These patients were older (67.9 vs 58.5 years) and more often lived alone (46.2\% vs $7.0 \%)$.

Conclusion: Patients that were discharged to rehab shared similar characteristics to those in previously published reports in arthroplasty and spine literature. Patients discharged to inpatient rehab were more likely to be older, have higher a higher body mass index (BMI), live alone, and undergo larger operations. Higher estimated blood loss (EBL), longer surgical time, and more levels fused, were all associated with discharge to rehab. Our scoring tool (PDAT) uses preoperative living situation, assistive device (AD) use, number of levels fused, and ASA classification to preoperatively predict discharge destination. It has similar predictive properties to the RAPT, which is currently used in patients undergoing hip or knee arthroplasty. Patient preference for a discharge to rehab should be taken into consideration as well.

Keywords: Discharge Disposition; RAPT; Discharge Tool; Scoring Tool; Predicting Discharge; Preoperative Assessment 


\section{Abbreviations}

ASA: American Society of Anesthesiologists Physical Status Classification; AD: Assistive Device; BMI: Body Mass Index; COPD: Chronic Obstructive Pulmonary Disease; EBL: Estimated Blood Loss; IRB: Institutional Review Board; LOS: Length of Stay; OT: Occupational Therapy; PT: Physical Therapy; RAPT: Risk Assessment and Predictor Tool; SNF: Skilled Nursing Facility

\section{Introduction}

Rates of lumbar fusion in the United States have risen 2.7-fold in recent years, from 77,682 cases in 1998 to 210,407 cases in 2008 [1]. During the same time, the total hospital charges associated with spinal fusion have increased from $\$ 24,676$ to $\$ 81,960$ and the national bill increased nearly 8-fold, from $\$ 4.3$ billion to $\$ 33.9$ billion [1]. Based on data from the Healthcare Cost and Utilization Project, the increase in number of spine fusions and cost exceed those of other common elective inpatient orthopedic procedures such as total hip and knee replacements [1]. Disposition after inpatient orthopedic surgery has an effect on total cost and may play a role in patient outcome and satisfaction. There is a paucity of literature identifying patients at risk for being discharged to a short-term inpatient rehabilitation facility or skilled nursing facility (SNF) after elective spine surgery. Determining early in the postoperative period or even preoperatively which patients will be most likely to need to be discharged to an inpatient facility has the potential to decrease length of stay and thereby decrease the total cost of admission. Early planning may also serve to mitigate patient expectations and improve satisfaction for those discharged directly home.

All patients undergoing lumbar spine fusion at our institution over a one-year period were reviewed and we identified preoperative risk factors unique to patients undergoing lumbar fusion that were discharged to an inpatient rehab. Our goal was to develop a scoring tool specific to patients undergoing lumbar fusion surgery to predict discharge disposition.

\section{Materials and Methods}

A retrospective chart review of 300 patients undergoing a lumbar spine fusion between January 1, 2014 and December 31, 2014 at our institution was performed. All operations were performed on an elective basis by six fellowship-trained orthopedic spine surgeons. Twelve patients were excluded for incomplete data. Institutional review board (IRB) approval was obtained.
Each patient's living situation, preference for discharge destination, and primary insurance payor were recorded from preoperative intake forms. Preoperative opioid use was documented in the preoperative medication reconciliation. BMI and number of medical comorbidities (including hypertension, diabetes, coronary artery disease, asthma/chronic obstructive pulmonary disease (COPD), renal insufficiency) were recorded from the preoperative history and physical. Operative records were reviewed to obtain the number of levels fused, surgical approach, EBL, operative time, and ASA for each patient. The number of physical therapy (PT) and occupational therapy (OT) sessions, preoperative use of AD such as a cane or walker and LOS were gathered from inpatient physical therapy and case management notes. If a patient underwent a planned staged procedure with two hospital admissions, each admission was counted individually since the discharge destination and operative data frequently varied between each stage.

\section{Statistical analysis}

All statistical analyses were performed using SPSS Version 22 (IBM Corp. Released 2013. IBM SPSS Statistics for Windows, Version 22.0. Armonk, NY: IBM Corp.). Each variable was subjected to chi-square, Fisher's exact test, independent t-test, or Wilcoxon rank-sum test to determine which variables were significantly associated with discharge destination. Surgical time and EBL were long-tailed right and thus log-transformation was used to recover normality prior to t-test. Logistic regression models were used to determine the weighted significance of each variable and develop the scoring tool.

\section{Results}

Out of the 300 patients undergoing lumbar fusion in 2014, 227 (76\%) were discharged directly home and 73 (24\%) were discharged to an inpatient rehab or skilled nursing facility. Median length of stay was $3 \pm 1$ days for all patients. Patients discharged to rehab had a significantly longer LOS (4.63 days) compared to those discharged home ( 4.63 days vs 2.88 days, $\mathrm{p}<0.05$ ). Number of OT sessions and preoperative use of opioids were not significantly different between patients discharged home and rehab $(p=0.098, p$ $=0.57$, respectively). Surgical approach data did not meet the expected cell count recommendation for chi-square testing due to the small number of anterior and lateral approaches in the sample and thus no significant association of surgical approach with discharge destination could be found. All other variables including age, sex, BMI, primary insurance payor, discharge preference, living alone, 
AD use, number of medical comorbidities, ASA classification, surgical time, EBL, number of fusion levels, number of PT sessions, and LOS were significantly different between patients discharged directly home and those discharged to rehab (Table 1).

\begin{tabular}{|c|c|c|c|}
\hline Variable & $\begin{array}{c}\text { Home } \\
(n=227)\end{array}$ & $\begin{array}{c}\text { Rehab } \\
(n=73)\end{array}$ & $\begin{array}{c}\text { p-val- } \\
\text { ue* }\end{array}$ \\
\hline Age (years) & $55.98 \pm 12.039$ & $68.27 \pm 8.805$ & 0.000 \\
\hline Sex (Female) & $44.1 \%$ & $63.0 \%$ & 0.007 \\
\hline $\begin{array}{l}\text { Body Mass Index } \\
\left(\mathrm{BMI}, \mathrm{kg} / \mathrm{m}^{2}\right)\end{array}$ & $28.98 \pm 5.09$ & $30.77 \pm 6.64$ & 0.036 \\
\hline $\begin{array}{l}\text { Comorbidities } \\
(\geq 2)\end{array}$ & $16.3 \%$ & $38.4 \%$ & 0.001 \\
\hline $\begin{array}{l}\text { Surgical time } \\
\text { (minutes) }\end{array}$ & $181.4 \pm 70.75$ & $255.5 \pm 113.33$ & 0.000 \\
\hline $\begin{array}{l}\text { Estimated Blood } \\
\text { Loss (mL) }\end{array}$ & $237.4 \pm 222.85$ & $458.8 \pm 509.92$ & 0.000 \\
\hline $\begin{array}{l}\text { Physical Therapy } \\
\text { (PT) sessions }\end{array}$ & $2.41 \pm 1.21$ & $3.64 \pm 1.89$ & 0.000 \\
\hline $\begin{array}{l}\text { Occupational } \\
\text { Therapy (OT) ses- } \\
\text { sions }\end{array}$ & $1.69 \pm 0.89$ & $1.58 \pm 1.38$ & 0.098 \\
\hline $\begin{array}{l}\text { Length of Stay } \\
\text { (days) }\end{array}$ & $2.88 \pm 1.58$ & $4.63 \pm 2.34$ & 0.000 \\
\hline $\begin{array}{l}\text { Preoperative opi- } \\
\text { oids (yes) }\end{array}$ & $34.4 \%$ & $38.4 \%$ & 0.57 \\
\hline $\begin{array}{l}\text { Private insurance } \\
\text { (yes) }\end{array}$ & $62.8 \%$ & $37.0 \%$ & 0.000 \\
\hline $\begin{array}{l}\text { Discharge prefer- } \\
\text { ence (prefer } \\
\text { rehab) }\end{array}$ & $0.4 \%$ & $19.4 \%$ & 0.000 \\
\hline $\mathrm{ASA} \geq 3$ & $15.9 \%$ & $47.9 \%$ & 0.000 \\
\hline Levels fused $\geq 4$ & $3.5 \%$ & $24.7 \%$ & 0.000 \\
\hline Live alone (yes) & $5.3 \%$ & $20.0 \%$ & 0.000 \\
\hline AD use (yes) & $16.7 \%$ & $65.3 \%$ & 0.000 \\
\hline \multicolumn{3}{|l|}{ Approach* } & \multirow{5}{*}{0.101} \\
\hline Posterior & $67.4 \%$ & $74.0 \%$ & \\
\hline Lateral & $3.1 \%$ & $2.7 \%$ & \\
\hline Anterior & $7.9 \%$ & $0.0 \%$ & \\
\hline Combined & $21.6 \%$ & $23.3 \%$ & \\
\hline
\end{tabular}

Table 1: Univariate testing to determine which variables significantly differed between those discharged home and those discharged to rehab.

*: Greater than $80 \%$ of expected cell counts were less than 5 , so recoded as binary variable: posterior versus other approach.
Variables that were significant in the univariate analyses were then used to create a logistic regression model for predicting discharge destination. Because the goal was to create a tool that would allow predictions to be made prior to surgery, only the variables that could be collected preoperatively were included in the predictive model. Surgical time, EBL, PT sessions, and LOS were excluded on this basis. Further selection of variables to include in the PDAT was based on a balance of predictive accuracy with simplicity and ease of use. Sex and primary insurance payor were eliminated because no improvement in predictive accuracy was gained from these variables. Age and BMI were excluded because these variables added additional complexity to the scoring tool without improving predictive accuracy. Number of medical comorbidities closely correlated with ASA score.

The variables included in the final logistic regression model were $\geq 4$ levels fused, ASA of $\geq 3$, living alone and AD use (Table 2 ). Four of our 300 patients had a single missing data point in one of these categories and were excluded from the logistic regression analysis. The overall model was statistically significant with $\mathrm{p}<$ 0.05 . For a given data point, the regression model calculates the probability of discharge to rehab facility based on the values of the various predictors entered into the model. A single-unit increase in the value of any given predictor is associated with a change in the odds of discharge to rehab, reported as an odds ratio (Table 2). For example, patients who had 4 or more vertebral levels fused had nearly 7 times the odds of discharge to rehab compared to patients who had less than 4 levels fused.

\begin{tabular}{|l|c|c|c|}
\hline Predictor & $\begin{array}{c}\text { Odds Ratio } \\
\text { (OR) }\end{array}$ & $\begin{array}{c}\text { 95\% Confidence } \\
\text { Interval (CI) }\end{array}$ & p-value \\
\hline Levels fused $\geq 4$ & 6.901 & $2.420-19.681$ & 0.000 \\
\hline $\mathrm{ASA}^{ \pm} \geq 3$ & 3.163 & $1.586-6.308$ & 0.001 \\
\hline Live alone & 4.125 & $1.506-11.302$ & 0.006 \\
\hline $\mathrm{AD}^{*}$ use & 6.922 & $3.576-13.400$ & 0.000 \\
\hline
\end{tabular}

Table 2: Logistic regression model including variables that are known prior to surgery.

${ }^{ \pm}$: American Society of Anesthesiologists physical status classification (ASA); *: Assistive Device (AD).

Based on the slopes of the individual variables included in the model, a simplified scoring tool was developed to predict discharge outcome. The slope of each variable is proportional to the relative 
influence of that variable on predictions made by the logistic regression model. This proportionality is reflected in the weighted value assigned to each predictor within the PDAT scoring tool (Table 3). Two points are assigned for AD use and four or more levels fused, one point for living alone and ASA $\geq$ three. Scores can range from zero to six points, with higher numbers indicating greater likelihood of discharge to rehab.

\begin{tabular}{|l|c|c|}
\hline Question & Answer & Score \\
\hline 1) How many vertebral levels fused? & $\begin{array}{c}\text { 4 or more } \\
=2 \\
\text { 3 or less }\end{array}$ & $=0$ \\
\hline 2) What is the patient's ASA score? & $\begin{array}{c}\text { 3 or more } \\
=1\end{array}$ & $=0$ \\
\hline 3) Does the patient live alone? & Yes less & $=1$ \\
& No & $=0$ \\
\hline $\begin{array}{l}\text { 4) Does the patient use an assistance } \\
\text { device for walking? }\end{array}$ & Yes & $=2$ \\
& No & $=0$ \\
\hline
\end{tabular}

Table 3: Preoperative discharge assessment tool.

To test the accuracy of our scoring tool, it was used to create a new logistic regression model with each patient's score indicating the sole predictor of discharge destination. If the model calculated greater than $50 \%$ probability of discharge to rehab for a given data point, the model predicts rehab as the discharge destination of that individual. If the probability of discharge to rehab is calculated to be less than $50 \%$, the model predicts discharge to home. Within our dataset, we were able to predict discharge destination with $85.5 \%$ accuracy overall. Predictions of home discharge were $95.2 \%$ correct, which can be compared to $74.3 \%$ accuracy if we assumed that all patients within the sample would be discharged to home. Predictions of discharge to rehab were $53.6 \%$ accurate, which can be compared to $25.7 \%$ accuracy if we assumed that all patients within the sample would be discharged to rehab (Table 4).

\begin{tabular}{|l|c|c|c|}
\hline \multirow{2}{*}{ Observed } & \multicolumn{2}{|c|}{ Predicted } & \multirow{2}{*}{ Correct (\%) } \\
\cline { 2 - 4 } & Home & Rehab & \\
\hline Home & 214 & 13 & $94.3 \%$ \\
\hline Rehab & 30 & 39 & $56.5 \%$ \\
\hline Overall Percentage & \multicolumn{2}{|l}{} & $85.5 \%$ \\
\hline
\end{tabular}

Table 4: Predictive accuracy of logistic regression model.
Breakdown of the scores by range provides an indication of how this tool could potentially be used in clinical practice. 88\% of patients who scored 4 or more points are discharged to rehab, while $87 \%$ of patients who scored 2 or less points are discharged to home. If discharge to rehab is predicted for all patients with scores of 3 or greater, this prediction is accurate for $77 \%$ of cases (Table 5).

\begin{tabular}{|l|c|c|c|}
\hline \multirow{2}{*}{$\begin{array}{l}\text { Score } \\
\text { Group }\end{array}$} & \multicolumn{2}{|c|}{ Actual Discharge Destination } & \multirow{2}{*}{ Correct (\%) } \\
\cline { 2 - 3 } & Home (n) & Rehab (n) & \\
\hline$>3$ & 2 & 15 & $88.2 \%$ (rehab) \\
\hline$\geq 3$ & 11 & 37 & $77.1 \%$ (rehab) \\
\hline$<3$ & 216 & 32 & $87.1 \%$ (home) \\
\hline
\end{tabular}

Table 5: Predictive accuracy of scoring tool by score range.

One unique group of patients are those who requested to be discharged to an inpatient rehab facility after surgery (Table 6). These patients tended to be older, live alone, and use an AD preoperatively compared to those who were discharged directly home. Fourteen of the fifteen patients who requested to go to inpatient rehab were discharged to an inpatient facility.

\begin{tabular}{|l|c|c|c|}
\hline & $\begin{array}{c}\text { Requested } \\
\text { Rehab } \\
\text { N=15 }\end{array}$ & $\begin{array}{c}\text { Requested } \\
\text { Home } \\
\text { N= 284 }\end{array}$ & p-value \\
\hline Average Age (years) & 67.9 & 58.5 & 0.004 \\
\hline Live alone & $46.2 \%$ & $7.0 \%$ & 0.03 \\
\hline $\begin{array}{l}\text { Preoperative assistive } \\
\text { device use }\end{array}$ & $73.3 \%$ & $25.8 \%$ & 0.001 \\
\hline Discharge to rehab & $14(93.3 \%)$ & $58(20.4 \%)$ & 0.000 \\
\hline
\end{tabular}

Table 6: Discharge preference.

\section{Discussion}

Oldmeadow., et al. developed the Risk Assessment and Predictor Tool (RAPT) in 2003 to preoperatively identify patients that are high risk for discharge to an inpatient rehab facility after hip or knee replacement [2]. RAPT scores are used by many institutions, including our own, to help predict and facilitate safe disposition of patients from the hospital after total hip or knee arthroplasty. Since the original RAPT design, there have been several other tools developed to help anticipate and identify patients that may need inpatient rehab or ICU level monitoring post operatively. In the arthroplasty literature PLAN, PARS, and arthro-MAP have all been 
described [3-5]. Bekelis., et al. reviewed the National Surgical Quality Improvement Program (NSQIP) database to estimate the risk of postoperative complications after spine surgery [6]. Scoring tools will never guarantee complete accuracy, but they do serve to initiate communication between patients, their surgeon, and case managers (PLAN), or consulting medical services (PARS, arthroMAP, NSQIP) [3-6].

By understanding the applicability and shortcomings of RAPT, we can better understand the utility of our scoring tool. The RAPT was originally developed and validated on a cohort of Australian patients undergoing hip or knee replacement from 1998 - 2000. In this group, the average length of stay was 9.3 days for those discharged home and 10.6 days for those discharged to rehab. It was $89 \%$ accurate in this population for predicting discharge to rehab facility in the high-risk group. Overall accuracy was $83 \%$, which is similar to the overall accuracy of PDAT [2]. One potential limitation of the RAPT is its applicability to other patient populations and healthcare systems. Another is its relevance, with more streamlined postoperative protocols and a much shorter average length of stay after total joint arthroplasty.

Tan., et al. found that in a group of 569 patients, low RAPT score (high risk patients) correlated with discharge to rehab and a longer length of stay after total joint arthroplasty [7]. Hansen., et al. tested the predictive accuracy of the RAPT on over three thousand US patients undergoing total hip or knee replacement at an academic medical center from 2006 - 2011 [8]. The authors found the overall predictive accuracy of RAPT within their patient population to be $78 \%$. They also determined the predictive accuracy of each individual score and found the lowest accuracy $(65 \%)$ in patients scoring 7 - 10 points, where nearly $50 \%$ of their patients scored. According to their analysis, the intermediate risk group should be defined by scores of $7-10$, rather than $6-9$ [8]. One of the confounders the authors discussed was insurance status, which may play more of a role in discharge destination in the US than in countries such as Australia with equal access to free healthcare and supplemental private insurance [8]. In our cohort, patients discharged to rehab were more likely to have Medicare as their primary payor. Ultimately, insurance status did not add to the predictive accuracy of our scoring tool and was excluded.

Our cohort of patients demonstrated patterns that are consistent with those in previously published literature. Increasing age, increased BMI, and number of medical comorbidities are all patient factors associated with discharge to rehab. Patients with private insurance were discharged home more frequently than those with public insurance. This is consistent with previous studies in both the spine and arthroplasty literature [8,9]. In a multivariate analysis of factors affecting length of stay after lumbar fusion, Gruskay., et al. found that increasing age, ASA score, and discharge to inpatient rehab facility or SNF were factors associated with a significantly longer hospital stay after lumbar spine fusion [9]. History of cardiac disease was associated with a decreased length of stay, which the authors state may be related to a more extensive preoperative workup and medical management while in the hospital [9]. Intraoperative factors such as higher EBL, OR time, and number of levels fused were also associated with discharge to rehab, which is also consistent with previous studies [10,11]. Similar to the original RAPT study, patients who use assistive devices for ambulation or who live alone were also more likely to be discharged to rehab [2].

Discharge preference was the most heavily weighted variable with an odds ratio greater than 30 , nearly 5 times greater than the odds ratio of any other variable. Fifteen of the 300 patients in our sample stated that they preferred discharge to rehab. The average age of these patients is significantly greater than the average age of patients who preferred discharge to home (67.9 and 58.5 years, respectively). In addition, those patients who prefer rehab are more likely to live alone ( $46.2 \%$ versus $7.0 \%$ ) and more likely to use an assistance device when walking (73.3\% versus $25.8 \%$ ). In our sample, 14 out of 15 (93.3\%) patients who preferred to be discharged to a rehab facility were ultimately discharged to inpatient rehab. Rehab preference is a subjective measure susceptible to influence by care providers as well as by other objective variables included in the model. We thought it better to treat this variable separately from the rest of the model to better capture the effect of the other more objective variables on discharge outcome. Patient preference for discharge to rehab should still be considered a strong indicator of ultimate discharge destination.

Surgical approach may have an influence on discharge destination, but we did not have enough patients undergoing isolated anterior and lateral approaches to show significance. We have developed a unique predictive scoring tool that can be used to facilitate patient discharge after lumbar spine fusion. Ideally, the predictive accuracy of the scoring tool should be tested prospectively on ad-

Citation: Ashley Rogerson., et al. "Preoperative Discharge Assessment Tool (PDAT): Predicting Disposition After Lumbar Spine Fusion". Acta Scientific Orthopaedics 4.8 (2021): 39-44. 
ditional samples in the future to determine the external validity of the tool.

\section{Conclusion}

Our scoring tool (PDAT) uses preoperative living situation, AD use, number of levels fused, and ASA classification to preoperatively predict discharge destination. It has similar predictive properties to the RAPT, which is currently used in patients undergoing hip or knee arthroplasty and this scoring tool may be utilized for patients undergoing lumbar spine fusion surgery.

\section{Bibliography}

1. Rajee SS., et al. "Spinal fusion in the United States: analysis of trends from 1998-2008". Spine 37.1 (2012): 67-76.

2. Oldmeadow., et al. "Predicting risk of extended inpatient rehabilitation after hip or knee arthroplasty". The Journal of Arthroplasty 18.6 (2003): 775-779.

3. Barsoum W., et al. "Predicting patient discharge disposition after total joint arthroplasty in the United States". The Journal of Arthroplasty 25.6 (2010): 885-892.

4. Courtney PM., et al. "Predictors of the Need for Critical Care After Total Joint Arthroplasty: An Update of Our Institutional Risk Stratification Model". The Journal of Arthroplasty 29 (2014): 1350-1354.

5. Weurz TH., et al. "A nomogran to predict major complications after hip and knee arthroplasty". The Journal of Arthroplasty 29 (2014): 1457-1462.

6. Bekelis K., et al. "A predictive model of complications after spine surgery: the National Surgical Quality Improvement Program (NSQIP) 2005-2010". The Spine Journal 14 (2014): 1247-1255.

7. Tan C., et al. "Predicting discharge outcomes after total knee replacement using the Risk Assessment and Predictor Tool". Physiotherapy 100 (2014): 176-181.

8. Hansen VJ., et al. "Does the Risk Assessment and Prediction Tool predict discharge destination after total joint arthroplasty?" Clinical Orthopaedics and Related Research 473 (2015): 597-601.

9. Gruskay JA., et al. "Factors affecting length of stay after elective posterior lumbar spine surgery: a multivariate analysis". The Spine Journal 15.6 (2013): 1188-1195.
10. Munin., et al. "Predicting discharge outcome after elective hip and knee arthroplasty". American Journal of Physical Medicine and Rehabilitation 74.4 (1995): 294-301.

11. Zheng F., et al. "Factors predicting hospital stay, operative time, blood loss and transfusion in patients undergoing revision posterior lumbar spine decompression, fusion and segmental instrumentation". Spine 27.8 (2002): 818-824.

\section{Volume 4 Issue 8 August 2021}

(c) All rights are reserved by Ashley Rogerson., et al. 